\title{
Design of Interoperability Checking Sequences Against WAP
}

\author{
O. Kone and J-P. Thomesse
}

LORIA - INPL, Campus Scientique B.P. 239 Vandoeuvre-les-Nancy 54506 France.

kone@loria.fr, thomesse@loria.fr

Key words: WAP, Application and Service Design, Design of Interoperability Tests.

Abstract: The deployment of a telecommunication service involves technical issues such as cellular transmission, routing etc, which are transparent to the user. Electronic mail or Short Message System are good examples of communication services used by persons provided with wireless terminals. The incoming generation of mobile equipment will access internet-like services and WAP (Wireless Application Protocol) is a standard destined to specify the related new architectures. WAP forum efforts will also concentrate on the development of tests aimed to insure the conformance and the introperability of WAP implementations. The contribution of this paper is the design of checking sequences for the interoperability of WAP implementations. Our work mainly contributes to phase two of WAP conformance process review.

\section{INTRODUCTION}

Many research works have been taken in various fields of wireless communication to solve technical issues such as cellular transmission and routing $[9,18,14]$, application and services [8]. The dynamism in wireless communication is due to the permanent evolution of mobile technology. For instance, the new generation of mobile equipment will not be restricted to classical telephony applications. More than standard communication services, internet-like services will be also accessible from mobiles.

"... The world's largest consumer payments organization and the world's number one mobile phone manufacturer have signed a global agreement to develop ways in which financial institutions and mobile 
phone operators can offer secure payment services to their customers via a mobile phone. The organizations will carry out joint market development activities and pilot technical payment alternatives ..."

WAP forum News, San Francisco, 8 February 2000.

The previous annoucement shows that the availability of Internet-like services from mobile terminals is becoming a reality. WAP (Wireless Application Protocol[16]) is a standard destinated to specify the concerned new architectures. The design of WAP systems will follow the classical protocol development cycle including specification, implementation and testing. A wide range of implementations belonging to different manufacturers will be available on the market. These implementations will face problems such as reliability, conformance/certification and interoperability (due to implementations heterogeneity).

Conformance testing consists of checking wether a given implementation meets the requirements of the standard specification $[7,11]$. Interoperability testing consists of checking wether different implementations successfully communicate and interwork according to the standard. WAP forum has started to define a testing framework which will be followed in two steps. The first step is concerned with conformance testing of WAP application part and will issue certification labels for WAP products. The second step is concerned with the interoperability of WAP layer-to-layer products and is also referred to as compliance testing.

The contribution of this paper is the design of checking sequences for the interoperability of WAP implementations. The proposed sequences are test pattern which can show that different client and server entities can interoperate or not.

In the following section, we recall the main features of WAP architecture, and we introduce the methodology we adopted for testing WAP. Section 3 describes our experiment in modelling and selection of test patterns for WAP.

\section{A METHODOLOGY TO TEST WAP INTEROPERABILITY}

\subsection{ARCHITECTURE OF WAP NETWORKS AND SERVICES}

WAP is aimed at enabling mobile terminals to access Internet-like services. Within the WWW (World Wide Web), a computer program can ask another one to execute some request. The first program is called client and the second one is called server. In order to insure interoperability, the WWW is built over standards such as HTTP [1] 


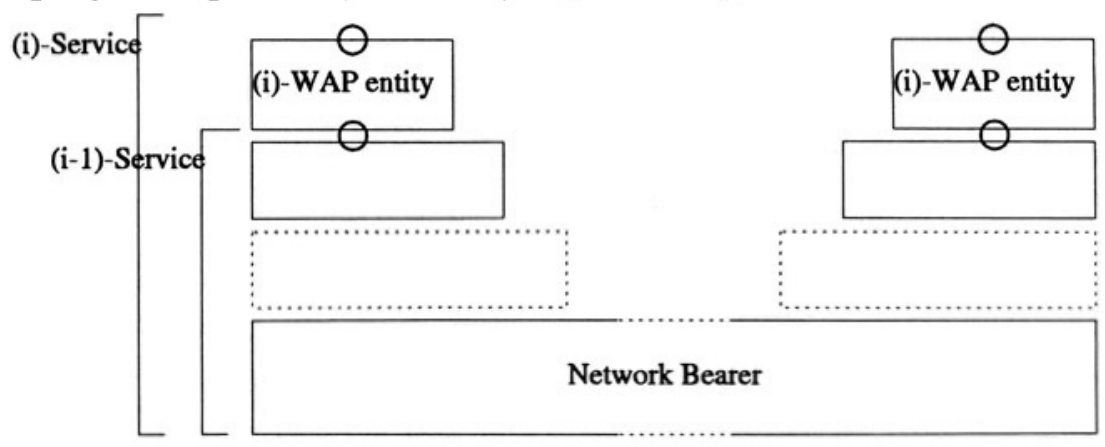

Figure 1 Structure of WAP services

and HTML [2]. The WWW model has influenced the WAP model very much. Distributed wireless applications are also composed of clients and servers. Mobile clients will have access to both WAP-origin and WWW servers. A WAP-origin server is, for example, a Wireless Telephony Application server which is a direct access point of a given wireless network provider to the WAP-client. But the communication with a WWW server requires to go through a WAP-proxy server which stands for an intermediary node between the two technologies. The mobile application will be programmed with WML (Wireless Markup Language) which is very close to HTML. The mobile terminal will be provided with a microbrowser for WML. WAP functionnalities will be carried out by a family of protocols that constitute the WAP-stack.

The structure of WAP model (figure 1) resembles the one of OSI basic reference model. An (i)-WAP layer is composed of (i)-WAP entities using a (i-1)-WAP service. One of the particularities of WAP structure is that some (i)-service can be directly used by upper layers or applications. For example, the Transaction layer or further defined applications can directly access the Transport layer.

Normative references tell more about WAP. The reader may report to the standards for detailed information.

\subsection{A FRAMEWORK TO DISTRIBUTED/INTEROPERABILITY TESTING}

This section describes the main characteristics of the methodology adopted for WAP tests development. The so-called ToP methodol- 
ogy $[10,12,13]$ has been a contribution to normative testing area for the interoperability of cornmunicating systems. Most experiments with TOP have been taken with standards such as ATM Adaptation Layer, ATM-ABR or OSI protocols $[3,4]$.

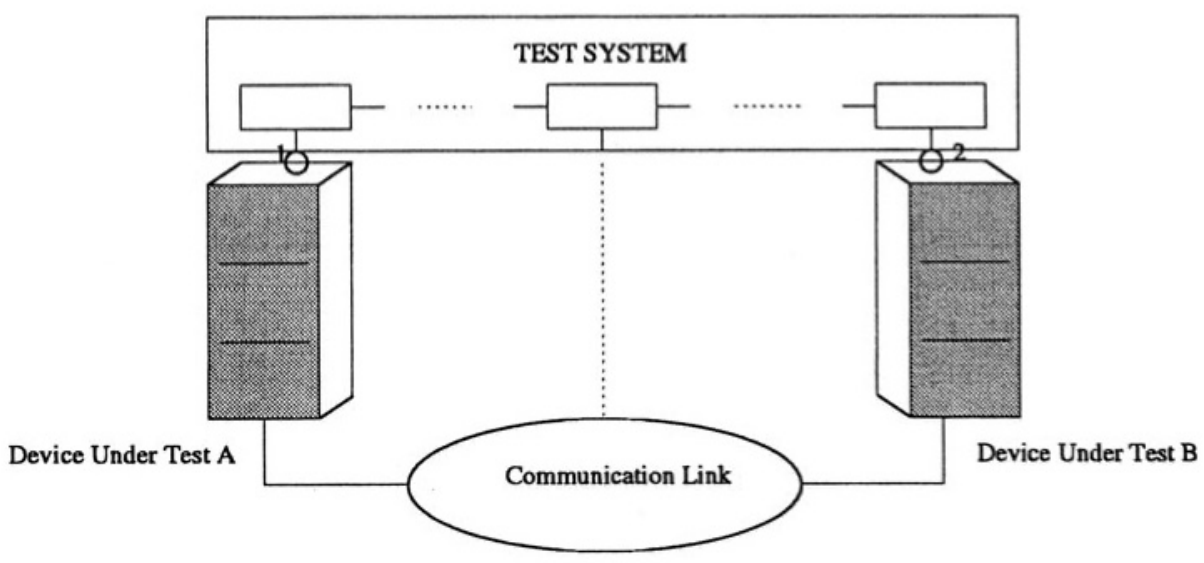

Figure 2 Interoperability Testing Architecture

Figure 2 depicts an abstract view of the Top test architecture. The Service Acces Points (SAP) are labeled with 1 and 2. The test system interacts with the devices under test through these SAP. An optional interaction point enables it to access the PDU (Protocol Data Unit) at lower levels.

Behavioural testing. The test campaign consists of subrnitting interactions to implementations while observing their reaction. Test sequences (or checking sequences) can be automatically computed if formal models of reference specifications are available. Our approach works with input/output automata for modelling the specifications and the test purposes ( $\stackrel{\text { def }}{=}$ the properties to be checked). The algorithm implemented in the TOP test generation tool is an on the fly seacrh [12] which explores the specifications of the devices under test and computes pertinent test pattern which can be used to experiment the implementations. More details on the ToP methodology and its applications are available in $[10,13]$.

Using ToP against WAP. Compare figures 1 and 2. As explained before, each WAP layer is intended for providing some service. This 
service is to be used by actual or further upper layers and applications. Obviously, the reliability of a given layer depends on the one of the underlying services. So checking the correctness of the service provided by implementations is fundamantal. The WAP architecture enables access to each layer of the WAP stack and the TOP architecture is powerful to check a distributed system through its service boundary. In practice, the connection to the service access points of the devices may require a few intrumentation. Typically, an interface may be used to encode/decode the interactions (or signals) exchanged between the test system and the devices under test. In figure 2, the white part of the devices represents such possible interfaces, while the grey part represent their internal design.

Moreover, WAP specifications include, for each layer, the protocol description by state tables. We modeled the specifications (client entity, server entity) with finite input/output automata which were further input to our test generation tool. The next section tells more about tests design for WAP.

\section{EXPERIMENT WITH THE DESIGN OF CHECKING SEQUENCES}

Because of the recursive structure of WAP service layers, ((i)-Service $\equiv(\mathrm{i})$-Protocole + (i-1)-Service), our work can be applied to each WAP layer. But in this paper, we will focus on our experiment with the WAP session layer (WSP), which is the top of the layers underlying the WAP Application Environment.

\subsection{MODELLING ISSUES}

WSP enables client and server applications to exchange contents through a maintained communication session. WSP specifications [17] include a connectionless protocol over a datagram service, and a connectionmode protocol over a transaction service. In the sequel, we consider the connection-mode protocol only, as the other one is fairly simple.

WSP is mainly featured to establish and release a session between a client and a server, exchange contents of the two applications, suspend and resume the session.

The WSP is defined over a set of service primitives and their ordering by time sequence charts. A service primitive is of the form S-Service-type, (e.g. S-Connect-req), where $S$ is the label of the session layer, Service indicates the name of the service (e.g. Connect). The type (e.g. request) is also indicated. Protocol operations are performed through the use of a transaction (TR) service. The PDU exchanged between the WSP peer- 
entities are encapsulated in the TR primitives: TR-Service-PDU denotes the encapsulation of a given PDU in a TR-Service primitive. For example, TR-Invoke-Connect represents the encapsulation of the Connect PDU in TR-Invoke. The session protocol is described with a set of state tables. We modeled the client and the server protocol entities with Input Output automata as this model was the formalism used by our test generation tool. In order to check the correctness of our model we translated it in SDL language [5] so that it could be verified with the ObjectGeode simulator/debugger. The specification file contained about 2500 lines of SDL code. Of course, the verification of the model revealed some errors of modelling (some transitions were forgotten) and mainly some unused transitions. It appeared that the unused transitions corresponded to the reaction of entities face to the possible errors introduced by the underlying layer. In fact, for the time being, our test design methodology does not deal with error generation which falls in the category of robustness testing. Error cases due to the communication environment are various and not controllable. Our test design process is based on automatic computation of the behaviour part assuming a normal operating environment. Further studies will investigate error generation. Another specification issue concerns optional services (e.g the Push service). Optional services are not systematically included in our reference model. We described these services as SDL processes which may be added or not to the mandatory part of the specification.

\subsection{TEST/CHECKING SEQUENCES}

In the WSP specifications, implementation features are organised in groups of functionality (Session creation, Session suspend/resume, Push facilities etc). We used the guide of WAP Implementation Conformance Statement [15] as a basis to test selection. Even if test patterns are produced automatically with our tool, test purposes are to be defined formally before, and this work is done by hands. In this paper, we will not include all of the test purposes considered. We will present two examples to illustrate the experiment with WSP layer : The session creation feature and the connection redirection.

\section{Session Creation. Connecting the mobile terminal}

Session creation starts with an S-Connect-req (Connection Request), and ends with an S-Connect-cnf (Connection confirmation). The session creation abstract behaviour can be defined with the test purpose below described with a finite automaton. The syntax expresses one transition per line. Each line describes an interaction as well as the implementation which executes it. For example, "1" represents the identi- 
fier of the WAP-client implemenpation (first and last lines), "2" represents the identifier of the WAP-server implemenpation (second and third lines).

TP1 ? 1.S-Connect-req 1 TP2

TP2 ! 2.S-Connect-ind 2 TP3

TP3 ? 2.S-Connect-res 2 TP4

TP4 ! 1.S-Connect-cnf 1 TP5

Our test generation tool computes the client and server WAP-specifications according to the test purpose. The tool produces a test pattern which can be used to experiment the expected feature (in this example, the connection establishment).

The lines of the form $\langle\mathrm{XXX} \mathrm{xxX} \mathrm{xXX}\rangle$ indicate the global state which may be actually reached by the distributed system: In the first line example, NULL represents the starting state of the client, the second NULL represents the starting state of the server, and TP1 is the beginning of the behaviour to be checked. Between global states are displayed the interaction to be executed.

<NULL NULL TP1>

1: ? 1.S-Connect-req 1

<NUCIA1 NULL TP2>

2: ! TR-Invoke-Connect 1 <CONNECTING NUCIA1 TP2>

3: ! TR-Invoke-ack 2 <CONNECTING NUCIA2 TP2>

4: ! 2.S-Connect-ind 2 <CONNECTING CONNECTING TP3>

5: ? 2.S-Connect-res 2 <CONNECTING CIC2A1 TP4>

6: ! TR-Result-ConnectReply 2 <CICEA1 CONNECTING-2 TP4>

7: ! TR-Result-ack 1 <CICEA2 CONNECTED TP4>

8: ! 1.S-Connect-cnf 1 <CONNECTED CONNECTED TP5>

1: A Connection request is received by implementation 1 (the client session entity).

2: The client invokes the transaction layer for sending its Connect PDU. 
3: Upon reception of that $\mathrm{PDU}$, the server (session entity) acknowledges it through the transaction layer.

4: The server sends a Connection indication to the upper layers.

5: Then it receives the response notifying that the application accepted the connection.

6: This notification is sent to the client, as a result of its request.

7: The client acknowledges the result.

8: The client session user is sent a confirmation indicating that the connection has been established.

Connection Redirection. Connection redirected by WAP server

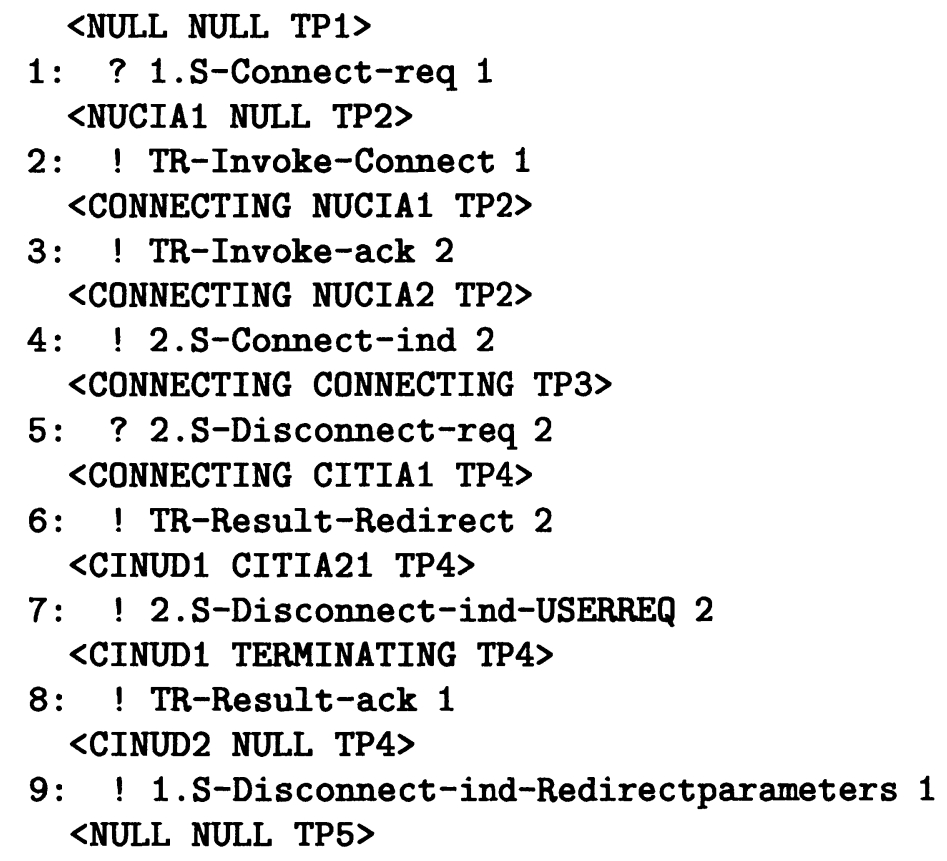

If a server can not respond (service unavailable or service moved), the connection may be redirected. In such case, the server session layer refuses the connection (line 5:) and a Redirect PDU is sent back to the client (line 6:) which may further try another connection.

The full execution of such test scenario by WAP client and server entities show their aptitude to interoperate. The occurrence of an unexpected interaction reveals an error. 


\section{CONCLUSION}

Manufacturers and service providers pay high for tests development. Because of the competition involved in the industry of wireless communication, the reliability of products (and their correctness according to standards) has become a design criterion of first choice. Testing is a well known means of satisfying such criterion. But the quality of a test pattern also depends on the quality of the test production process. The approach used in this paper is based on automatic tests computation, which is a good way to insure tests soundness and test reproductibility (New standards are always emerging, so one can not imagine to design test by hands all the time). Our work is a contribution to the development of WAP standard destinated to new wireless communication systems. For space reasons we cannot include all the test patterns designed for WAP. All the mandatory functionalities have been experimented in dynamic testing. But we did not complete optional aspects. For instance, we considered the Method Invocation facility, but not for all the methods. On the one hand, most of these methods are optional. On the other hand, test design for methods is a repetitive work as it follows the same scheme. Only the reference of the method changes. As WAP is concerned with a very new technology, the related specifications are subject to modifications and this may influence the test patterns defined somehow. The test patterns will be stable when the standard will be definitively adopted and widely implemented during the incoming months.

\section{References}

[1] Hypertext Transfer Protocol. HTTP/1.1, RFC2068. R.Fielding et al. January, 1997.

[2] HTML 4.0 Specification, W3C Recommendation REC-HTML40971218. D.Raggett et al. September 1997.

[3] ITU-T Recommandation Q.2110: B-ISDN ATM Adaptation Layer ITU Telecommunication Standard Sector 1994.

[4] Information Processing Systems. Open Systems Interconnection. Transport protocol International Standard ISO 8073, 1988 (F)AFNOR.

[5] ITU-T Recommandation Z.100: Specification and Description Language SDL, Contribution Com X-R215-E, 1987.

[6] ISO/TC 97/SC 16/WG1 IS 7498. Basic Reference Model for Open System Interconnection. 1983. 
[7] ISO/IEC 9646, Information Technology - Open Systems Interconnection - Conformance testing methodology and framework-Part 1-5.

[8] Veikko Hara, Jarmo Harju, Jouni Ikonen, Jari Porras. Application of Distributed Workstation Environment to the Parallel Simulation of Mobile Networks IFIP TC 6 - Task Group Wireless Communications. 2nd PWC workshop Frankfurt am Main, Germany, December 1996

[9] Juntong Liu and Gerald Q. Maguire Jr. GMRM: An Efficient Routing Model for an Integrated Wireless Mobile Packet Switch Network. The 3rd Workshop on Personal Wireless Communication (PWC98), Tokyo, Japan, 1998.

[10] O.Koné, R.Castanet. Test generation for interworking systems Computer Communications, Elsevier Science Publishers Vol. 23 N.7 Mars 2000. pp 642-652.

[11] O.Koné, R.Castanet. Including Physical Time Constraints into Conformance. Proc. IFIP symposium on Protocol Specification, Testing and Verification. Warsaw, Poland, June 1995.

[12] O.Koné, R.Castanet, P.Laurençot. On the fly test generation for real time protocols. Proc. International Conference on Computer Communication and Networks. Louisianne, USA. October 1998.

[13] O.Koné. The ToP Methodology for the Interoperability of communicating systems. Technical Report INPL, Nancy December 1999.

[14] Tsuyoshi Tamaki, Hitoshi Aida, Tadao Saito. A Reserved Channel Scheme of Dynamic Channel Assignment for Multimedia Mobile Communication s Systems. IFIP TC 6 - Task Group Wireless Communications 2nd PWC workshop. Frankfurt am Main, Germany, December 1996.

[15] Wireless Application Protocol. Conformance Statement, Compliance Profile and Release List. WAP forum, April 1998.

URL: http: //www . wapforum. com.

[16] Wireless Application Protocol. Architecture Specification, WAP forum, April, 1998.

URL: http://www . wapforum. com

[17] Wireless Session Protocol Specification. WAP forum, URL: http://www . wapforum. com, 1999

[18] Jzef Wozniak. Analysis of a New Hybrid PRMA-Type Channel Access Scheme with Frequency Hopping for Cellular Mobile Systems. IFIP TC 6 - Task Group Wireless Communications 2nd PWC Workshop. Frankfurt am Main, Germany, December 1996. 